\title{
The Mink Circovirus Capsid Subunit Expressed by Recombinant Baculovirus Protects Minks against Refractory Diarrhea in Field
}

\author{
Lidong Wang, Yanyan Zhang, Teng Chen, Lijuan Mi, Xuefei Sun, Xintao Zhou, Faming Miao, Shoufeng Zhang, \\ Ye Liu and Rongliang $\mathrm{Hu}$ *D
}

check for updates

Citation: Wang, L.; Zhang, Y.; Chen, T.; Mi, L.; Sun, X.; Zhou, X.; Miao, F.; Zhang, S.; Liu, Y.; Hu, R. The Mink Circovirus Capsid Subunit Expressed by Recombinant Baculovirus Protects Minks against Refractory Diarrhea in Field. Viruses 2021, 13, 606. https:// doi.org/10.3390/v13040606

Academic Editors:

Kyoko Tsukiyama-Kohara and Michinori Kohara

Received: 4 March 2021

Accepted: 28 March 2021

Published: 1 April 2021

Publisher's Note: MDPI stays neutral with regard to jurisdictional claims in published maps and institutional affiliations.

Copyright: (c) 2021 by the authors. Licensee MDPI, Basel, Switzerland. This article is an open access article distributed under the terms and conditions of the Creative Commons Attribution (CC BY) license (https:// creativecommons.org/licenses/by/ $4.0 /)$.
Laboratory of Epidemiology, Institute of Military Veterinary Medicine, Academy of Military Medical Sciences, Academy of Military Sciences, Jilin 130122, China; 15164390097@163.com (L.W.);

Yanyanzhang90615@163.com (Y.Z.); ctcx1991@163.com (T.C.); mlj84321@163.com (L.M.); sunxuefei0322@163.com (X.S.); zhouxtao@foxmail.com (X.Z.); miaofaming81@163.com (F.M.); zhangshoufeng@hotmail.com (S.Z.); liuye79@126.com (Y.L.)

* Correspondence: ronglianghu@hotmail.com; Tel.: +86-431-8698-5867

\begin{abstract}
Mink refractory diarrhea is a seasonal disease that occurs in many mink farms in China. Mink circovirus (MiCV) has been recognized as the causative agent of the disease. The aim of the study was to develop a subunit vaccine against mink refractory diarrhea. A recombinant baculovirus strain expressing the capsid protein was constructed using the baculovirus expression vector system (BEVS). A subunit vaccine was developed based on the capsid protein with appropriate adjuvant. Then, a field trial was carried out in two districts in order to evaluate the efficiency of the subunit vaccine. The field trial indicated that in total, only $1.8 \%$ of the minks developed typical diarrhea in the vaccinated group compared with $74.5 \%$ in the control group. The vaccination could significantly reduce the infection rate of $\mathrm{MiCV}$ among the mink herds and could restrain the virus' shedding from feces. Furthermore, the vaccinated group had a higher average litter size in the following year compared to the control group. Collectively, the results indicated that the subunit vaccine based on the capsid protein can provide reliable protection against MiCV infection.
\end{abstract}

Keywords: mink circovirus; capsid subunit; recombinant baculovirus; field vaccination

\section{Introduction}

Mink refractory diarrhea, also called "autumn diarrhea" or "red and grey diarrhea" by local farmers, has appeared in mink farms in China since the mid-1980s. In a typical outbreak, usually 70-80\% of minks on a farm develop diarrhea, anorexia, and poor fur quality, and $7-8 \%$ die as a result [1]. Due to the considerable economic loss caused by the disease, an autogenous vaccine, which was made using formalin-inactivated gut tissue suspension of the infected minks, was initially put into application. This strategy indeed reduced the morbidity and partially controlled the disease for a period of time around the 2000s. However, after a few years of application, the vaccinated pedigree breeds appeared to show symptoms of Aleutian mink disease. Given the high infection rate of the Aleutian mink disease virus ( $>60 \%$ ) among mink herds in China [2], it can be speculated that the autogenous vaccine contains inactivated Aleutian mink disease viruses that enhance immune complex formation and lead to the Aleutian mink disease [3]. Hence, the autogenous vaccine has been abandoned in recent years, and there is an urgent need for a safe and efficient vaccine to control the disease.

Mink circovirus (MiCV) was first discovered in mink farms in Dalian, China, in 2013 and has been recognized as the etiological agent of mink refractory diarrhea $[1,4]$. MiCV belongs to the genus circovirus of the family Circoviridae and it is composed of a capsid protein and a circular, single-stranded genome that contains two open reading frames (ORFs), named ORF1 (rep gene, 894 bp) and ORF2 (cap gene, 684 bp) [5]. Phylogenetic 
analysis of the cap gene showed that MiCV was genetically closest to the bat circovirus, with a sequence similarity of about $73 \%$ [6]. For the members of circovirus, the capsid protein is usually considered to play a key role in the immunoprotection against infection. For instance, one dose of a porcine circovirus type 2 subunit vaccine based on the capsid protein demonstrated the ability to induce both a humoral and cellular immune response, thus reducing the morbidity, mortality rate, viremia, and viral load under the field trial [7].

The baculovirus expression vector system (BEVS) is a kind of eukaryotic expression system. Since it was initially used for the production of human $\beta$-interferon successfully in 1983 [8], BEVS made a great contribution to the development of veterinary subunit vaccines. The first globally available commercial veterinary vaccine based on BEVS was the E2 subunit vaccine against classical swine fever. Two weeks after vaccination, pigs could significantly resist against the intranasal challenge with 100 median lethal dose $\left(\mathrm{LD}_{50}\right)$ of the virulent classical swine fever virus [9]. The above-mentioned porcine circovirus type 2 subunit vaccine is also based on the capsid protein expressed by BEVS. Furthermore, some other vaccines such as bluetongue virus vaccines [10-12] and avian influenza virus vaccines $[13,14]$ have also shown good immunogenicity, despite having no commercial application at present.

In this study, we aimed to utilize the BEVS to express the capsid protein of MiCV and develop a subunit vaccine against mink refractory diarrhea. Subsequently, we carried out a field trial in mink farms. The efficiency of the subunit vaccine was evaluated by a clinical symptom observation, fecal sample test, and average litter size analysis.

\section{Materials and Methods}

\subsection{Generation and Production of Recombinant Baculoviruses}

A Bac-to-Bac baculovirus expression system (Life Technologies, Carlsbad, CA, USA) was used for the generation and production of recombinant baculoviruses. MiCV-DL13 (an MiCV strain first discovered in Dalian, China, in 2013) (NC_023885.1) samples were derived from the livers and intestines of infected minks and were kept frozen at $-80{ }^{\circ} \mathrm{C}$. DNA was extracted from the MiCV samples using a viral DNA extraction kit (Corning, Wujiang, China). The capsid gene was cloned using a polymerase chain reaction (PCR) approach with primers containing homologous ends of the pFastBacI transfer plasmid (forward: $5^{\prime}$ CGCGGCCGCTTTCGAATCTAGAATGCCCGTAAGATCGCGATACT $-3^{\prime}$, and reverse: 5' ACTTCTCGACAAGCTTGGTACCTTAGTGATGGTGATGGTGATGAGTTTGCTTTGGG AAATTG $-3^{\prime}-$ the underlined sequences at $5^{\prime}$ were the homologous ends of the pFastBacI transfer plasmid and the underlined sequence in the middle of the reverse primer was the sequence of the $6 \times$ His fusion tag). The $\mathrm{pFastBacI}$ vector was linearized using restriction enzymes (Xba I and Kpn I) (New England Biolabs, Beijing, China) and the encoding sequence of the capsid protein with the C-terminal $6 \times$ His fusion tag was seamlessly inserted via external homologous recombination with an in-fusion cloning kit (Takara, Beijing, China) following the manufacturer's protocol. The recombinant clones were transferred into Escherichia coli DH5 $\alpha$ (Takara, Beijing, China) for amplification and were extracted with a plasmid miniprep kit (Corning, Wujiang, China). The recombinant plasmids were verified using PCR with pFastBacI vector primers (forward: 5'-CTCCGGAATATTAATAGATC $-3^{\prime}$, and reverse: $5^{\prime}$-CAAATGTGGTATGGCTGATT $-3^{\prime}$ ). The recombinant transfer vectors were sequenced to ensure that no mutation occurred.

The recombinant transfer vectors were transformed into Escherichia coli DH10Bac competent cells (Biomed, Beijing, China) and incubated on agar plates containing $50 \mu \mathrm{g} / \mathrm{mL}$ kanamycin, $7 \mu \mathrm{g} / \mathrm{mL}$ gentamicin, $10 \mu \mathrm{g} / \mathrm{mL}$ tetracycline, $100 \mu \mathrm{g} / \mathrm{mL}$ 5-Bromo-4-chloro-3indolyl $\beta$-D-galactopyranoside (X-gal), and $40 \mu \mathrm{g} / \mathrm{mL}$ isopropyl-beta-D-thiogalactopy ranoside (IPTG) (Takara, Beijing, China) for $48 \mathrm{~h}$ at $37^{\circ} \mathrm{C}$. The white clones were selected and amplified in liquid culture containing $50 \mu \mathrm{g} / \mathrm{mL}$ kanamycin, $7 \mu \mathrm{g} / \mathrm{mL}$ gentamicin, and $10 \mu \mathrm{g} / \mathrm{mL}$ tetracycline. Then, the recombinant bacmids were extracted using alkaline lysis, described elsewhere [15], from the bacteria and were further verified using PCR with M13 primers (forward: 5'-CCCAGTCACGACGTTGTAAAACG-3', and reverse: 5'- 
AGCGGATAACAATTTCACACAGG-3'). The naked transfer vectors were transformed into Escherichia coli DH10Bac competent cells in the same way.

Sf9 cells (Life Technologies, Carlsbad, CA, USA) were transfected with recombinant bacmids. Confluency and viability were assessed using a trypan blue staining cell viability assay kit (Beyotime Biotechnology, Shanghai, China) following the manufacturer's instruction manual. Briefly, $2 \mu \mathrm{g}$ recombinant bacmids and $6 \mu \mathrm{L}$ transfection reagent (Mirus Bio, Madison, WI, USA) were incubated in $100 \mu \mathrm{L}$ Sf-900 II serum-free medium (Life technologies, Carlsbad, CA, USA) for about 5 min each and mixed gently. The mixture was incubated at room temperature for $20 \mathrm{~min}$ and was added dropwise to the culture of monolayer Sf9 cells at 70\% confluency. The transfected cells were cultured in Sf-900 II serum-free medium with $10 \%$ heat-inactivated fetal calf serum at $27{ }^{\circ} \mathrm{C}$ in a cabinet for 5 days to generate initial recombinant baculoviruses. The non-recombinant baculoviruses were generated from naked bacmids in the same way as the control.

\subsection{Expression of the Capsid Protein}

Sf9 cells at $90 \%$ confluency were infected with recombinant baculoviruses at a multiplicity of infection (MOI) of 0.1 and incubated in the dark at $27^{\circ} \mathrm{C}$ for the expression of capsid protein. Furthermore, High Five (H5) cells (Life Technologies, Carlsbad, CA, USA) were seeded by recombinant baculoviruses at the same MOI and were cultured in suspension in Sf-900 II serum-free medium at $27^{\circ} \mathrm{C}$ at the speed of $110 \mathrm{rpm} / \mathrm{min}$ for $72 \mathrm{~h}$ for rapid production of the capsid protein. In addition, Sf9 cells and $\mathrm{H} 5$ cells were both infected with non-recombinant baculoviruses in the same way as the control.

\subsection{PCR Identification of Recombinant Baculoviruses}

The $\mathrm{H} 5$ cells were harvested $72 \mathrm{~h}$ post-infection (pi). Then, genomic DNA was extracted from the cells using a viral DNA miniprep kit (Corning, Wujiang, China), following the manufacturer's instruction manual, and was identified using PCR with M13 primers (forward: $5^{\prime}$-CCCAGTCACGACGTTGTAAAACG $-3^{\prime}$, and reverse: $5^{\prime}$ - AGCGGATAACA ATTTCACACAGG $-3^{\prime}$ ) to verify the presence of the recombinant baculoviruses.

\subsection{Indirect Immunofluorescence Assay}

The Sf9 cell monolayer was gently washed twice with phosphate-buffered saline (PBS) $(\mathrm{pH}=7.4)$ and fixed with $80 \%$ cold acetone for $30 \mathrm{~min}$ at $4{ }^{\circ} \mathrm{C}$. Two kinds of antibodies, anti-His tag monoclonal antibody (CWBIO, Beijing, China) and tetramethylrhodamine isothiocyanate (TRITC)-conjugated goat anti-mouse immunoglobulin G (IgG) (CWBIO, Beijing, China), were used for an incubation with the cell monolayer for $1 \mathrm{~h}$ at $37^{\circ} \mathrm{C}$ successively. Then, the cell monolayer was washed three times with PBS with $0.05 \%$ Tween-20 (PBST) and was watched under a florescent microscope.

\subsection{Sodium Dodecyl Sulfate-Polyacrylamide Gel Electrophoresis (SDS-PAGE) and Western Blot Analysis}

The H5 cells were harvested $72 \mathrm{~h}$ pi and were washed three times with PBS, counted, and resuspended in PBS at a concentration of $2 \times 10^{6}$ cell equivalents per milliliter. The cells were frozen and thawed twice for lysis. The crude lysate was mixed with a sample buffer and boiled for $5 \mathrm{~min}$. After a simple centrifugation, the proteins in the supernatant were separated on polyacrylamide gel. After that, the polyacrylamide gel was stained using Coomassie brilliant blue solution for $2 \mathrm{~h}$ and was then de-stained for several hours for observation. In addition, another same polyacrylamide gel was transferred onto a polyvinylidene fluoride (PVDF) membrane (Bio-Rad, Berkeley, CA, USA). The membrane was blocked with $5 \%$ skim milk in PBS at $4{ }^{\circ} \mathrm{C}$ overnight and probed with an anti-His tag monoclonal antibody (CWBIO, Beijing, China). Then, the membrane was probed with a horseradish peroxidase (HRP)-conjugated goat anti-mouse IgG (CWBIO, Beijing, China). After being washed with PBST three times, the membrane was developed using tetramethylbenzidine (TMB) (Kirkegaard \& Perry Laboratories, Gaithersburg, MD, USA), a horseradish peroxidase substrate, according to the manufacturer's instructions. 


\subsection{Vaccine Preparation}

The $\mathrm{H} 5$ cells were harvested $72 \mathrm{~h}$ pi, counted, and prepared at a concentration of $2 \times 10^{6}$ cell equivalents per milliliter of cell culture. The cells were frozen and thawed twice for lysis. Then, $0.9 \mathrm{~mL}$ crude lysate from $\mathrm{H} 5$ cells infected by recombinant baculoviruses and $0.1 \mathrm{~mL} \mathrm{10 \%} \mathrm{aluminum} \mathrm{hydroxide} \mathrm{gel} \mathrm{(Thermo} \mathrm{Fisher} \mathrm{Scientific,} \mathrm{Waltham,} \mathrm{MA,} \mathrm{USA)}$ were fully mixed and stirred as one dose of subunit vaccine. For the control, $0.9 \mathrm{~mL}$ pure cell culture was mixed under the same condition. All of the preparations were stored on ice until inoculation.

\subsection{Animal Experimental Design}

We performed a field vaccination trial in selected mink farms in Zhuanghe, Liaoning, and Zhucheng, Shandong. The animals included in the trial were American minks (Neovison vison) (half male and half female) at about 4 months of age. The field trial began at the end of June in Zhuanghe, Liaoning, and at the end of August in Zhucheng, Shandong. In total, 2000 animals received one dose of the subunit vaccine $(1 \mathrm{~mL})$ intramuscularly as the vaccinated group (Vaccinated). Meanwhile, another 1000 animals received the equivalent control preparation (cell culture mixed with aluminum hydroxide gel) in the same way as the control group (Control). The experimental unit was a single mink in a single cage. Housing, husbandry, feeding, and other conditions were kept identical for both experimental groups in each district. The operators and keepers were blind to the experimental design. Table 1 lists the number of animals included in each group and in each district.

Table 1. The number of animals included in each group and each district.

\begin{tabular}{cccc}
\hline District & Vaccinated & Control & Total \\
\hline Zhuanghe & 800 & 400 & 1200 \\
Zhucheng & 1200 & 600 & 1800 \\
Total & 2000 & 1000 & 3000 \\
\hline
\end{tabular}

About two or three months after vaccination, from the end of September to the end of November, the most common time for mink refractory diarrhea, the two groups were clinically monitored daily for typical diarrhea.

Seventy fecal samples from each group in each district were randomly collected. The samples were fully mixed with tris (hydroxymethyl) aminomethane (Tris)-buffered saline and were centrifuged at a speed of $5000 \mathrm{r} / \mathrm{min}$ for $15 \mathrm{~min}$. The viral DNA in the supernatant was extracted using a viral DNA extraction kit (Corning, Wujiang, China) and was detected using PCR with primer pairs described elsewhere [16].

Around late April to early May of the following year, when the whelping season came, the number of pregnant minks and their offspring was recorded. Then, the average litter size of each group was calculated in order to evaluate the fecundity. The average litter size usually means the ratio of newborn offspring to previous pregnant animals in a group.

\subsection{Statistical Analysis}

The data of morbidity among mink herds and positive rate of the fecal samples were analyzed using the Pearson chi-square test. The data of the average litter size are presented as mean \pm SD values. Statistical analysis of the average litter size was performed by applying Student's $t$-test. $p$-values $<0.05$ were considered statistically significant. The statistical analysis was performed using IBM SPSS Statistics software (ver.19.0; IBM, Armonk, NY, USA).

\section{Results}

\subsection{The Capsid Protein of MiCV Was Successfully Expressed}

The recombinant baculoviruses were confirmed by PCR (Figure 1a). The indirect immunofluorescence assay showed that a specific bright orange-red image was captured in 
the Sf9 cells infected by recombinant baculoviruses, while there was no such image in the Sf9 cells infected by non-recombinant baculoviruses, nor in the uninfected cells (Figure 1b). (The results of identification of recombinant transfer vectors and bacmids are shown in Supplementary Figure S1.)

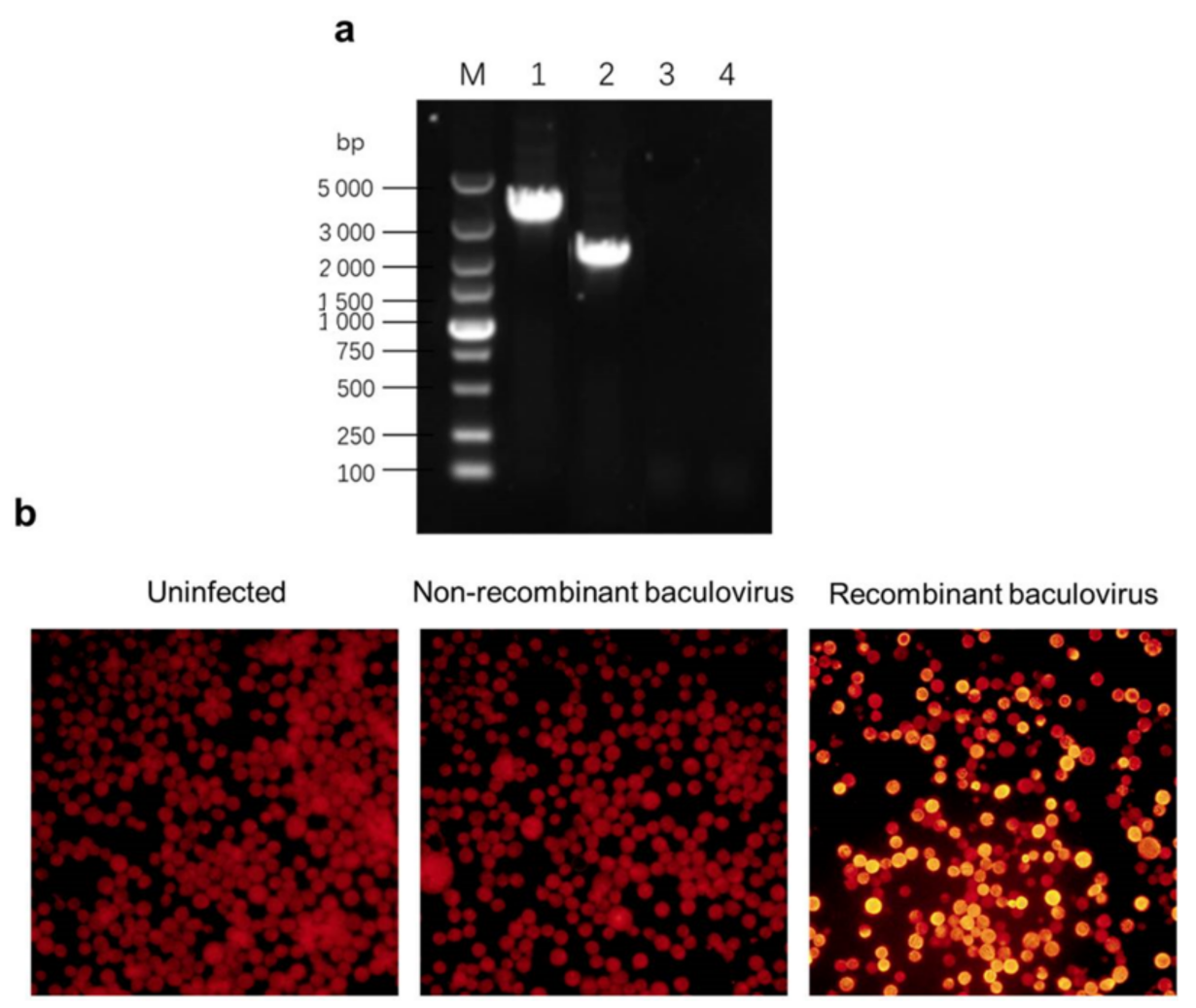

Figure 1. Agarose gel electrophoresis and fluorescence imaging analyses of recombinant baculoviruses. (a) Agarose gel electrophoresis analysis. Lane 1: Polymerase chain reaction (PCR) product of recombinant baculoviruses; lane 2: PCR product of non-recombinant baculoviruses; lane 3: PCR product of uninfected H5 cells; lane 4: negative control. (b) Fluorescence imaging analysis. Sf9 cells that were infected by non-recombinant or recombinant baculoviruses were detected under a fluorescent microscope. The stain was tetramethylrhodamine isothiocyanate (TRITC) and the original magnification was $100 \times$.

The Western blot analysis confirmed the presence of the capsid protein (Figure 2a). The SDS-PAGE analysis indicated that the molecular weight of the capsid protein was about $27 \mathrm{kDa}$ and the amount of capsid protein was obviously larger than that of other irrelevant proteins (Figure 2b).

a

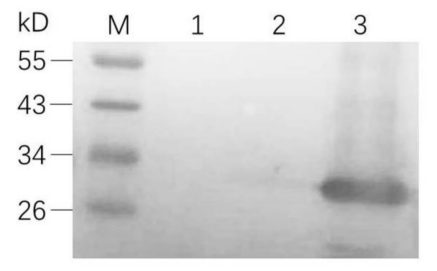

b

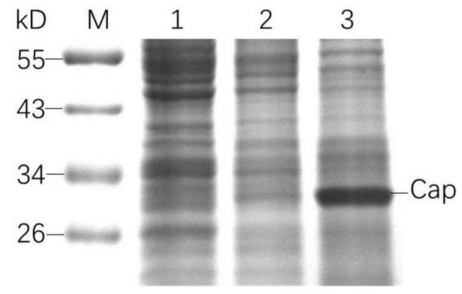

Figure 2. SDS-PAGE and Western blot analyses of the capsid protein. (a) Western blot analysis. (b) SDS-PAGE analysis showed the molecular weights of the capsid protein. Lane 1: Protein from crude lysate of uninfected $\mathrm{H} 5$ cells; lane 2: protein from crude lysate of $\mathrm{H} 5$ cells infected by nonrecombinant baculoviruses; lane 3: protein from crude lysate of $\mathrm{H} 5$ cells infected by recombinant baculoviruses. Cap: capsid protein. 


\subsection{Reduction in Morbidity and Infection Rate in Vaccinated Animals}

According to the observation of feces (the appearances of normal feces and typical diarrhea are shown in Figure 3), in total, only 36/2000 (1.8\%) minks developed typical diarrhea in the vaccinated groups compared to $745 / 1000(74.5 \%)$ in the control groups. The morbidity rates among the vaccinated groups were far lower than those of the control groups regardless of district or in total (Figure 4a).
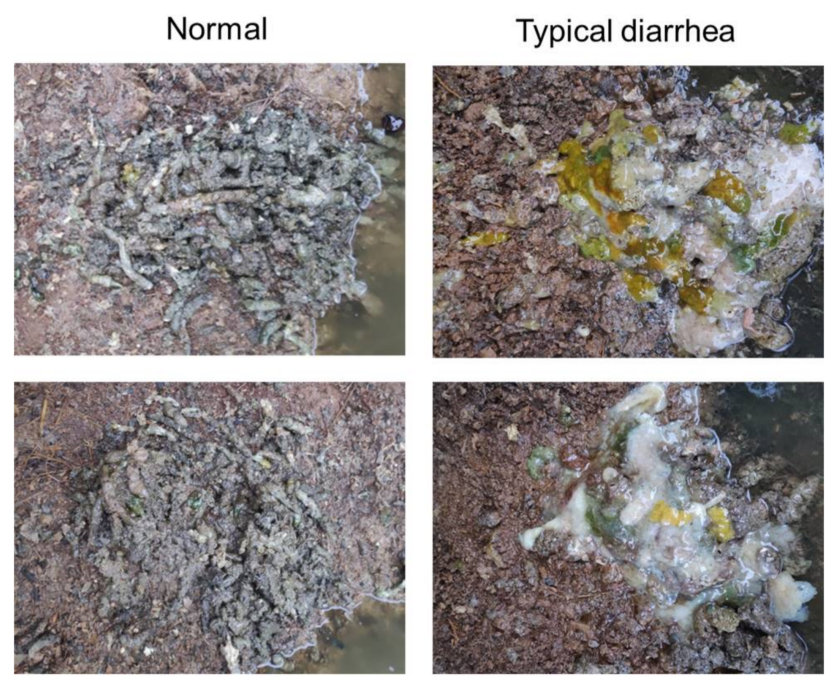

Figure 3. Appearances of normal feces and typical diarrhea. Two samples are displayed for each category. The feces of healthy minks were dark green and relatively dry (normal), while those of sick minks were yellow, grey, and waterish (typical diarrhea), which is the classical symptom of mink refractory diarrhea.

a

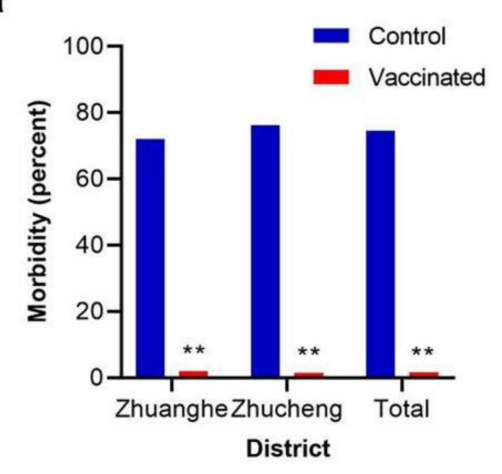

C

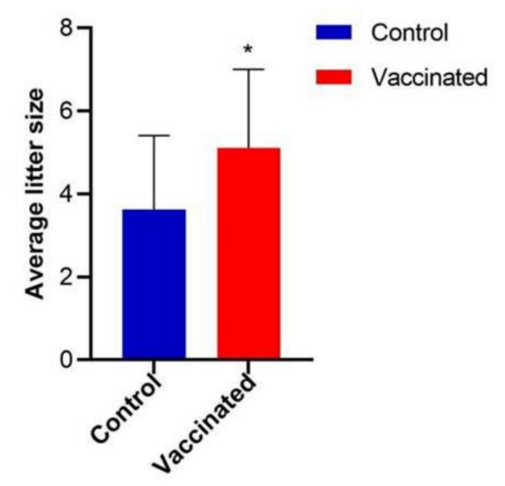

b

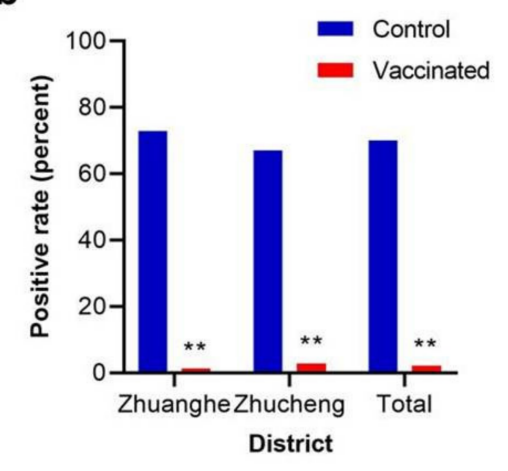

Figure 4. Comparison of morbidities, positive rates of fecal samples, and average litter sizes between control and vaccinated groups. (a) Comparison of morbidities between control and vaccinated groups in Zhuanghe, Zhucheng, and total. (b) Comparison of positive rates of fecal samples between control and vaccinated groups in Zhuanghe, Zhucheng, and total. (c) Comparison of average litter sizes between control and vaccinated groups in total $\left(* p<0.05\right.$; $\left.{ }^{* *} p<0.01\right)$. 
The PCR test of fecal samples showed that the infection rate of MiCV among the vaccinated groups was $2.1 \%$, while that of the control groups was $70.0 \%$. The infection rates among the vaccinated groups were obviously lower than those of the control groups regardless of district or in total (Figure $4 b$ ).

\subsection{Comparasion of Average Litter Size}

In total, 500 and 250 pregnant minks in the vaccinated and control groups were observed, respectively. The average litter size of the vaccinated groups in the following year was $5.11 \pm 1.89$, while that of the control groups was $3.62 \pm 1.78$ (Figure $4 \mathrm{c}$ ). The vaccinated group showed a higher fecundity.

\section{Discussion}

Circovirus has various hosts among mammals and birds. In addition to the wellknown porcine circovirus, circoviruses that can infect canines, penguins, bats, and other animals have been reported [17-19] in recent years. As a new circovirus member, MiCV has only been discovered in China and was even reported to be able to infect foxes and raccoon dogs [5]. Although $\mathrm{MiCV}$ can cause relatively large economic loss to the mink industry, few valid prevention technologies are available at present. Some new diagnostic techniques such as real-time quantitative PCR, indirect enzyme-linked immunosorbent assay, and recombinase polymerase amplification have been established [20-22]. However, vaccines against $\mathrm{MiCV}$ have not yet been developed. In this study, the subunit vaccine against MiCV was confirmed to be effective.

The capsid protein of MiCV has been previously expressed in the yeast system by the researchers in our laboratory [23]; nonetheless, the yield was too limited (less than $20 \mu \mathrm{g} / \mathrm{mL}$ ) for vaccine development. The potential for high-level recombinant protein production is one of the advantages of the BEVS, with "high-level" defined rather loosely as $\geq 100 \mu \mathrm{g}$ of recombinant protein per milliliter of infected insect cell culture [24]. In this study, the highest yield of the capsid protein reached $150 \mu \mathrm{g} / \mathrm{mL}$.

Up to now, MiCV has not been successfully cultured in any cell lines yet. Therefore, it is a challenge to evaluate the efficacy of vaccines considering the lack of efficient artificial infection methods. However, mink refractory diarrhea is a typical seasonal disease with classic symptoms that can be easily recognized. Furthermore, a previous investigation indicated that the disease occurs almost every year at a regular time, with relatively stable morbidity [1]. Therefore, we made the judgement that natural infection is a feasible method for the field trial. According to the experience of local farmers, there is a close relationship between the epidemic time and low temperature. This is the reason why the field trial started at different times in two districts. The infection rate of MiCV in mink farms in China was approximately $54.6 \%$ in 2014 [6], while in this study, the infection rate among control groups reached nearly $70 \%$, partially because the animals included in the field trial were all newborn in April or May of that year. Using younger animals avoided the potential influence of original antibodies because these animals had little chance of being infected by MiCV before the field trial. Given the instability of natural infection, a large number of animals were included in the field trial in order to obtain reliable data. However, as no artificial infection was included, the field trial did not inflict much additional suffering on the animals.

$\mathrm{MiCV}$ was reported to be able to cause a reduction in litter size and smaller newborn cubs [25]. In this study, we indeed confirmed this phenomenon. Usually, the highest average litter size of mink herds in China is about 5.50, and that of the vaccinated group in this study essentially reached the normal level, but 3.62 in the control group was, no doubt, a significant decline. Some other circoviruses, such as porcine circovirus type 2, can inflict reproductive failure, including abortions, stillbirths, etc. [26]. However, in addition to purely low litter size, we did not observe further reproductive failure symptoms as seen in porcine circovirus type 2 . The mechanism is poorly understood at present. 
During the field trial, no adverse effect of the vaccination was observed, which means that the vaccine causes no harm to the body. Due to the lack of a strict challenge experiment, the minimum dose of the vaccine has not been ascertained yet. In addition, the dose-effect relationship, the antibody titer, and other evaluation indexes of the subunit vaccine call for further research.

In summary, the capsid protein subunit vaccine was successfully constructed with BEVS and showed expectative effectiveness and safety. The field trial indicated that the subunit vaccine can reduce the morbidity, restrain the viruses shedding from the feces, and improve the fecundity. The subunit vaccine is expected to become a reliable biological product to contain MiCV infection in China.

Supplementary Materials: The following are available online at https:/ /www.mdpi.com/article/10 .3390/v13040606/s1, Figure S1: Identification of transfer vectors and recombinant bacmids.

Author Contributions: Conceptualization: R.H.; data curation: Y.Z.; formal analysis: L.W.; funding acquisition: R.H.; investigation: Y.L., L.W.; methodology: Y.Z., L.M., and X.S.; resources: R.H.; software: X.Z.; validation: R.H.; visualization: L.W.; supervision: S.Z.; project administration: R.H.; writing — original draft: L.W.; writing—review and editing: T.C., F.M., and R.H. All authors have read and agreed to the published version of the manuscript.

Funding: This research was funded by the National Natural Science Foundation of China, grant number 31472214.

Institutional Review Board Statement: All of the animals were treated in accordance with the provisions of the Welfare and Ethics of Laboratory Animals of China, and the protocol for this trial was approved by the Animal Welfare and Ethics Committee of the Institute of Military Veterinary Medicine, Academy of Military Medical Science (IACUC of AMMS-11-2018-001).

Informed Consent Statement: Not applicable.

Data Availability Statement: Not applicable.

Conflicts of Interest: The authors declare no conflict of interest.

\section{References}

1. Lian, H.; Liu, Y.; Li, N.; Wang, Y.; Zhang, S.; Hu, R. Novel Circovirus from Mink, China. Emerg. Infect. Dis. 2014, 20, 1547-1549. [CrossRef] [PubMed]

2. Gong, Q.-L.; Li, D.; Diao, N.-C.; Liu, Y.; Li, B.-Y.; Tian, T.; Ge, G.-Y.; Zhao, B.; Song, Y.-H.; Li, D.-L.; et al. Mink Aleutian disease seroprevalence in China during 1981-2017: A systematic review and meta-analysis. Microb. Pathog. 2020, 139, 103908. [CrossRef] [PubMed]

3. Porter, D.D.; Larsen, A.E.; Porter, H.G. Aleutian disease of mink. Adv. Immunol. 1980, 29, 261-286. [PubMed]

4. Ge, J.; Gu, S.; Cui, X.; Zhao, L.; Ma, D.; Shi, Y.; Wang, Y.; Lu, T.; Chen, H. Genomic characterization of circoviruses associated with acute gastroenteritis in minks in northeastern China. Arch. Virol. 2018, 163, 2727-2735. [CrossRef]

5. Yang, Y.; Cheng, Y.; Li, N.; Cheng, S.; Guo, L.; Zhou, Y.; Zhang, H.; Zhang, X.; Ren, L. Mink Circovirus Can Infect Minks, Foxes and Raccoon Dogs. Virol. Sin. 2018, 33, 561-564. [CrossRef]

6. Wang, Y.; Liu, Y.; Lian, H.; Li, N.; Zhang, L.; Hu, R. Molecular epidimiology of Mink circovirus. Chin. J. Vet. Med. 2015, 51, 6-8.

7. Martelli, P.; Ferrari, L.; Morganti, M.; De Angelis, E.; Bonilauri, P.; Guazzetti, S.; Caleffi, A.; Borghetti, P. One dose of a porcine circovirus 2 subunit vaccine induces humoral and cell-mediated immunity and protects against porcine circovirus-associated disease under field conditions. Vet. Microbiol. 2011, 149, 339-351. [CrossRef]

8. Smith, G.E.; Summers, M.D.; Fraser, M.J. Production of human beta interferon in insect cells infected with a baculovirus expression vector. Mol. Cell. Biol. 1983, 3, 2156-2165. [CrossRef]

9. Moormann, R.J.; Bouma, A.; Kramps, J.A.; Terpstra, C.; De Smit, H.J. Development of a classical swine fever subunit marker vaccine and companion diagnostic test. Vet. Microbiol. 2000, 73, 209-219. [CrossRef]

10. Stewart, M.; Bhatia, Y.; Athmaran, T.N.; Noad, R.; Gastaldi, C.; Dubois, E.; Russo, P.; Thiéry, R.; Sailleau, C.; Bréard, E.; et al. Validation of a novel approach for the rapid production of immunogenic virus-like particles for bluetongue virus. Vaccine 2010, 28, 3047-3054. [CrossRef]

11. Qiu, J.; Pérez de Diego, A.C.; Athmaram, T.N.; Stewart, M.; Rodríguez-Sánchez, B.; Sánchez-Vizcaíno, J.M.; Noad, R.; Roy, P. Characterization of Protection Afforded by a Bivalent Virus-Like Particle Vaccine against Bluetongue Virus Serotypes 1 and 4 in Sheep. PLoS ONE 2011, 6, e26666. 
12. Stewart, M.; Dubois, E.; Sailleau, C.; Bréard, E.; Viarouge, C.; Desprat, A.; Thiéry, R.; Zientara, S.; Roy, P. Bluetongue virus serotype 8 virus-like particles protect sheep against virulent virus infection as a single or multi-serotype cocktail immunogen. Vaccine 2013, 31, 553-558. [CrossRef]

13. Lee, D.-H.; Park, J.-K.; Lee, Y.-N.; Song, J.-M.; Kang, S.-M.; Lee, J.-B.; Park, S.-Y.; Choi, I.-S.; Song, C.-S. H9N2 avian influenza virus-like particle vaccine provides protective immunity and a strategy for the differentiation of infected from vaccinated animals. Vaccine 2011, 29, 4003-4007. [CrossRef]

14. Song, J.-M.; Choi, C.-W.; Kwon, S.-O.; Compans, R.W.; Kang, S.-M.; Kim, S.I. Proteomic Characterization of Influenza H5N1 Virus-like Particles and Their Protective Immunogenicity. J. Proteome Res. 2011, 10, 3450-3459. [CrossRef]

15. Sambrook, J.; Maniatis, T.; Fritsch, E.F. Molecular Cloning: A Laboratory Manual, 2nd ed.; CSH: New York, NY, USA, 1989 ; Volume 3.

16. Wang, Y.; Liu, Y.; Lian, H.; Li, N.; Hu, R.; Zhang, L. A PCR methods for detection of mink circovirus. Chin. J. Vet. Sci. 2015, 35, 1774-1776.

17. Matsumoto, T.; Sato, M.; Nishizono, A.; Ahmed, K. A novel bat-associated circovirus identified in northern Hokkaido, Japan. Arch. Virol. 2019, 164, 2179-2182. [CrossRef]

18. Morandini, V.; Dugger, K.M.; Ballard, G.; Elrod, M.; Schmidt, A.; Ruoppolo, V.; Lescroel, A.; Jongsomjit, D.; Massaro, M.; Pennycook, J.; et al. Identification of a Novel Adelie Penguin Circovirus at Cape Crozier (Ross Island, Antarctica). Viruses 2019, 11, 1088. [CrossRef]

19. Piewbang, C.; Jo, W.K.; Puff, C.; van der Vries, E.; Kesdangsakonwut, S.; Rungsipipat, A.; Kruppa, J.; Jung, K.; Baumgartner, W.; Techangamsuwan, S.; et al. Novel canine circovirus strains from Thailand: Evidence for genetic recombination. Sci. Rep. 2018, 8, 7524. [CrossRef]

20. Ge, J.; Shi, Y.; Cui, X.; Gu, S.; Zhao, L.; Chen, H. Rapid and sensitive detection of mink circovirus by recombinase polymerase amplification. J. Virol. Methods 2018, 256,1-5. [CrossRef]

21. Cui, X.; Shi, Y.; Zhao, L.; Gu, S.; Wei, C.; Yang, Y.; Wen, S.; Chen, H.; Ge, J. Application of Real-Time Quantitative PCR to Detect Mink Circovirus in Naturally and Experimentally Infected Minks. Front. Microbiol. 2018, 9, 937. [CrossRef]

22. Ge, J.; Cui, X.; Shi, Y.; Zhao, L.; Wei, C.; Wen, S.; Xia, S.; Chen, H. Development and application of an indirect enzyme-linked immunosorbent assay based on recombinant capsid protein for the detection of mink circovirus infection. BMC Vet. Res. 2018, 14, 29.

23. Zheng, G.; Lu, X.; Lian, H.; Zhang, S.; Liu, Y.; Hu, R. Secretotry expression of mink circovirus capsid protein in pichia pastoris. Chin. Vet. Sci. 2015, 45, 1266-1269.

24. Jarvis, D.L. Chapter 14 Baculovirus-Insect Cell Expression Systems. In Guide to Protein Purification, 2nd ed.; Burgess, R.R., Deutscher, M.P., Eds.; Elsevier Science \& Technology: Amsterdam, The Netherlands, 2009; Volume 463, pp. 191-222.

25. Sun, X.; Shao, Q.; Wang, Z. Dignosis and treatment of the disease caused by MiCV. Chin. Livest. Poult. Breed. 2015, 11, 131.

26. Meng, X.J. Porcine circovirus type 2 (PCV2): Pathogenesis and interaction with the immune system. Annu. Rev. Anim. Biosci. 2013, 1, 43-64. [CrossRef] [PubMed] 